\title{
Identification of Nocturnal Movements During Sleep using the Non- Contact Under Mattress Bed Sensor
}

\author{
Lorcan Walsh, Eddie Moloney, and Seán McLoone, Member, IEEE
}

\begin{abstract}
This paper describes the calculation of statistical, spatial and spatiotemporal features from a novel non-contact technology for sleep monitoring, the Under Mattress Bed Sensor (UMBS). Data was collected from two relatively healthy adults with a possible sleep disorder in a clinical setting. Methods for the extraction of statistical data describing overall bed restlessness, a spatial description of movement (centre and spread of pressure) and a spatiotemporal description of each in-bed body movement over the entire sleeping episode are discussed using the pressure sensing grid. These provide a quantitative description of sleep and restlessness throughout the night.
\end{abstract}

\section{INTRODUCTION}

$\mathbf{I}^{\mathrm{T}}$ has been found that sleep duration (for both long and short sleepers) is associated with an increased risk of death (relative risk of 1.3 and 1.12 respectively) [1]. It has also been shown that sleep disturbances may be indicative of poor health and functional deficits, especially in older adults $[2,3]$. Total sleep time is reduced in the elderly and this is not due to a reduced need for sleep, but in a diminished ability to sleep [4]. The NIH in the US has identified the need for a large scale screening and diagnoses methodology to evaluate the large population suspected of having sleep disorders [5]. Long term sleep monitoring has been shown to reflect changes in health status, such as those from serious life events and progression of an illness, specifically in older adults [6]. We propose a non-contact, ambient sleep monitoring technology, suitable for long-term deployment, which examines bed restlessness and sleeping patterns.

Polysomnography (PSG) is the gold standard sleep assessment technology and involves the recording of multiple physiological signals (including brain activity, muscle tone, eye movements, heart rate and respiration) during sleep. This is intrusive, costly, time consuming, often alienates the patient and is not suitable for large scale screening or diagnosis . Each 30-second epoch of sleep is classified by a trained scorer according to a strictly defined set of rules [7]. An inter-rater agreement rate of $82 \%$ has been reported using data from multiple subjects and across

Manuscript received March 26th, 2011. This work was supported in part by the Health Informatics Society of Ireland (HISI). The first author greatly acknowledges the financial support provided by The Irish Research Council for Science, Engineering and Technology under its Embark Initiative and Intel Ireland, Leixlip, Co. Kildare, Ireland and also the Postgraduate Travel Fund / Collins Kitchin Fund, NUI Maynooth.

Lorcan C. Walsh is a postgraduate researcher with the National University of Ireland (NUI) Maynooth; e-mail: lwalsh@eeng.nuim.ie.

Dr Eddie Moloney MD FRCPI FCCP, Consultant Respiratory Physician and Senior Lecturer in Medicine, Trinity College Dublin.

Dr. Seán F. McLoone PhD, Senior Lecturer and Head of Department with the Dept of Electronic Engineering, NUI Maynooth, Kildare, Ireland. separate sleep laboratories [8]. Wrist actigraphy is the current ambulatory gold standard accumulating the activity of the patient using a single metric over a pre-defined duration [9]. It has been shown to estimate nocturnal sleep duration and sleep-wake patterns reliably where PSG is not a suitable alternative. A low wake detection capacity is often reported with this device and accuracy is dependent on the active participation of the wearer. Sleep diaries are subjective questionnaires used (often concomitantly with wrist actigraphy) to estimate sleep duration, however their validity again relies on the participant. Each of these technologies has advantages and disadvantages, linked to the trade-off made between accuracy and long term deployment.

Indirect technologies offer a more ambient, practical approach to long term sleep monitoring (by avoiding conscious participation of the subject) and can include video and PIR based monitoring, radar based technologies, loadcell movement detection sensors and under mattress sensors.

We demonstrate the potential of the under mattress bed sensor (UMBS) in capturing nocturnal movement information such as a slow turn or a higher intensity posture shift. The UMBS consists of a grid of 24 fibre optic based pressure sensors, or tactels, integrated into a lightweight $1 \mathrm{~cm}$ x $90 \mathrm{~cm}$ x $23 \mathrm{~cm}$ foam mat. It was originally developed by Tactex Controls Inc. as a bed occupancy sensor. However the application of novel algorithms provides a description of in-bed movement throughout the sleeping episode. This system is an ideal ambient, pervasive sleep monitoring solution and a genuine competitor to wrist actigraphy [10].

This paper provides a validation of algorithms which extract movement-related features furthering previous discussion regarding its potential sleep monitoring application. Section II provides details of the technology, the data collection system and of the cohort. Section III discusses the extraction of statistical, spatial and spatiotemporal features from the UMBS data. The results are presented in Section IV and a brief discussion is given in Section V.

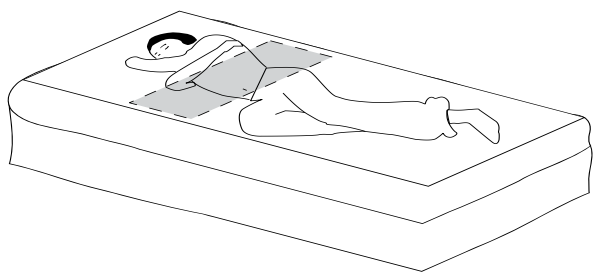

Figure 1. Typical Position of UMBS Relative to the Subject. In practice, it is placed beneath the mattress during recording. 

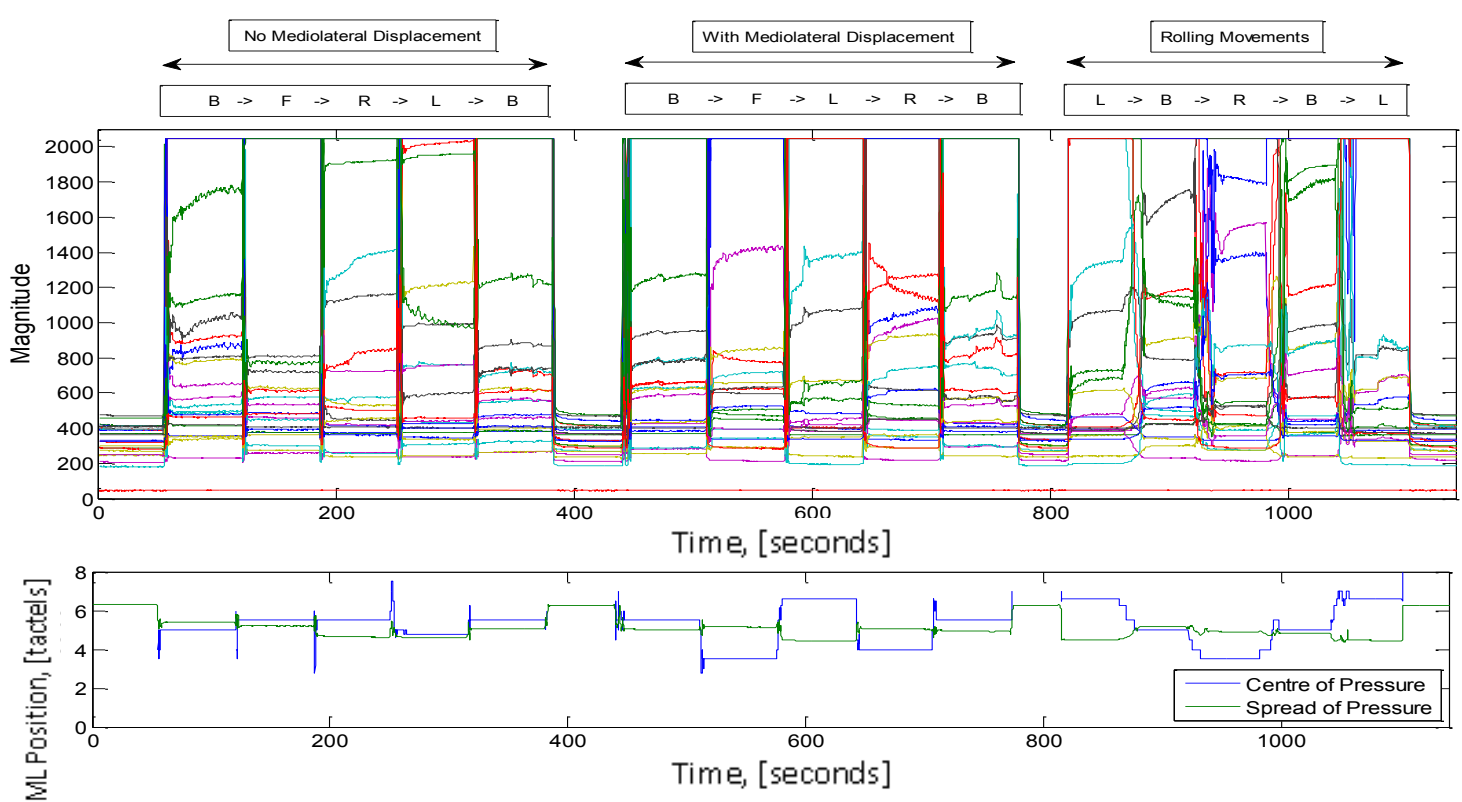

Figure 2. Example Bed Movement Data with the Posture of the Subject Changing from Lying on their Back (B), Front (F), Left (L) and Right (R) Sides. The change in the centre and spread of mediolateral (ML) position across the UMBS shows postural shifts.

\section{METHODS}

\section{A. UMBS and Data Collection System}

The UMBS pressure sensing grid was placed beneath the upper torso of the subject for the purpose of this data collection as shown in Figure 1. As the subject lies on the mattress, their body weight results in an increased pressure across the pressure sensing grid. Example data showing repeated postural shifts can be seen in Figure 2.

The pressure applied to each of the 24 individual pressure tactels are continuously refreshed internally within the UMBS at a rate of $20 \mathrm{~Hz}$. Data were polled from the UMBS using customized software run on a Dell Precision laptop with $2 \mathrm{~Gb}$ of RAM and an Intel CPU. Data were collected at a non-constant sampling (mean: $15.28 \mathrm{~Hz}$; range: $9.1 \mathrm{~Hz}-21.74 \mathrm{~Hz}$ ). This was due to a simplex polling protocol within the UMBS resulting in an inconsistent sampling rate. Linear interpolation was used to provide a constant sampling rate of $10 \mathrm{~Hz}$. Tactel values are nonlinear and could not be converted to absolute pressure values.

\section{B. Clinic-based UMBS Data Collection}

The sensor was deployed in a cohort of relatively healthy adults undergoing assessment for a potential sleep disorder at the Sleep Clinic in Peamount Hospital, Newcastle, Co. Kildare. This study was granted ethical approval by the Ethics Review Board at NUI Maynooth. Initial data from the two participants is analysed in this paper. Typical nocturnal UMBS data recorded over the first 233.3 minutes of the night for one participant can be seen in Figure 3.

\section{Typical Nocturnal Bed Movements}

The nocturnal UMBS movement patterns can be broadly classed into two separate categories: 1) large body movements, such as postural shifts and movement-related arousals, and 2) small body movements, such as those relating to breathing and heart rate. Data relating to large body movements are discussed in this paper. The large body movement patterns were further split into three categories; 1) quick postural shifts, 2) quick postural shifts with a corresponding mediolateral change in position across the bed and 3) slow rolling movements across the bed.
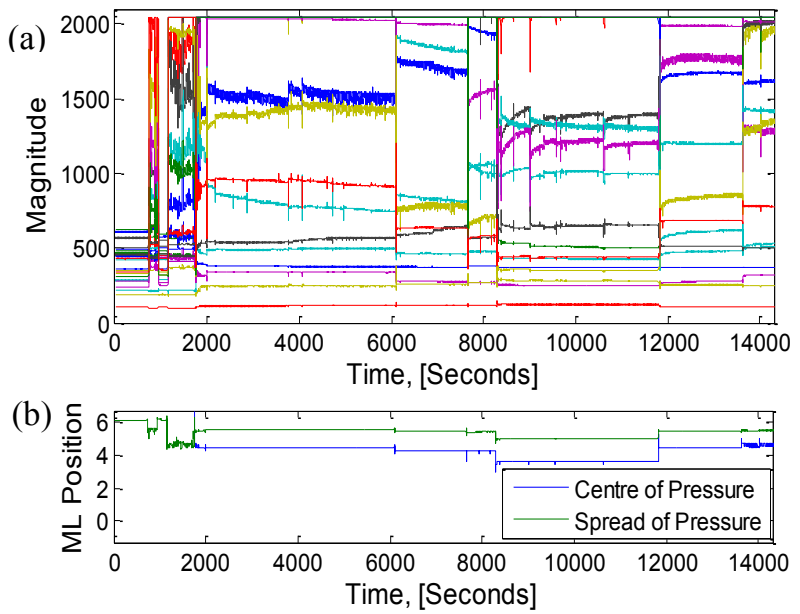

Figure 3. (a) Typical Nocturnal UMBS Data. (b) corresponding mediolateral shifts in the centre-point of pressure and spread of the pressure throughout the night.

\section{FEATURE EXTRACTION}

A flow chart describing the process of extracting the statistical, spatial and spatiotemporal features from the sensor data is given in Figure 5.

\section{A. Statistical Data}

Statistical UMBS features were derived to provide interand intra-daily comparisons of bed-restlessness. Data relating to general sleeping patterns can be derived from the 
UMBS. Such metrics include nocturnal restlessness, number and duration of bed exits, time in bed, settling time and sleep start/end times. Nocturnal restlessness was quantified using the derivative of the UMBS data. The UMBS derived motion metric was validated using 60 -seconds epochs in experimental conditions on 4 healthy adults against a webcam and a motion detecting algorithm [11]. The optimal movement detection threshold was selected empirically to provide high sensitivity and specificity rates. A previous comparison of UMBS motion metrics against wrist actigraphy has shown high agreement rates. Derived UMBS data has been shown to indicate bed restlessness and nocturnal rhythms in a community dwelling older adult population .

\section{B. Spatial Data}

Spatial UMBS features were derived to describe changes in the distribution of pressure across the sensor throughout the night. For each frame of data, the centroid of all 'active tactels- (CAAT), derived using an empirically defined threshold, was found over both the horizontal and vertical directions and defined as the 'centre of pressure'. The 'spread of pressure' across the UMBS was calculated by firstly reducing each frame of UMBS data to a one dimensional axis through a summation of the vertical tactels. This eight point axis was then linearly interpolated to contain 100 points. The spacing representing an eighty percent spread of pressure, beginning from the CAAT-point and spreading outwards, was calculated for each frame of UMBS data.

Slow rolling turns result in a slow shift in the centre of pressure and a relatively low magnitude of movement. However, sudden movements contain a large magnitude of movement, as shown in Figure 2. The spread of the data was proposed to distinguish between side-lying, supine or prone sleeping postures.

\section{Spatiotemporal Data}

Spatiotemporal UMBS features examined motion-in-bed and extracted magnitude, duration and medio-lateral displacement for each movement. Epochs deemed to contain motion, as defined in Section III (A), were analysed using an empirically defined refractory period to find the end of that motion . Low-pass filtering was applied to smoothen out the signal and remove any non-large-movement related artefacts, such as breathing. Subsequently movement was defined to have occurred if the difference between the current tactel value and the next over all of the 24 tactels exceeded an empirically defined threshold. Metrics derived for each movement included:

- The quantity of movement: the area under the curve during the movement; see Figure 4(b).

- The magnitude of movement: the quantity of movement normalised by the duration of that movement

- The displacement due to the movement: the mediolateral change in centre of pressure as a result of that movement.
- The time to the peak motion: the time difference between the movement start and the peak time of movement.

\section{RESULTS}

Example UMBS movement data is shown in Figure 2. However, these data are atypical as the movements are rapid (typically less than 5 seconds in duration) and the period between movements is short.

Example statistical, spatial and spatiotemporal features for two participants are given in Table 1. The statistical data describes the nocturnal restlessness of the participant expressed as a percentage of time in bed. This was further broken down empirically into small, medium and large movements. A spatial analysis of the UMBS data describes the medio-lateral displacement between samples during the recording period. The spatiotemporal data extracted from the UMBS provides quantitative statistics on individual nocturnal movements. The duration of such movements over a whole night for two participants is detailed.
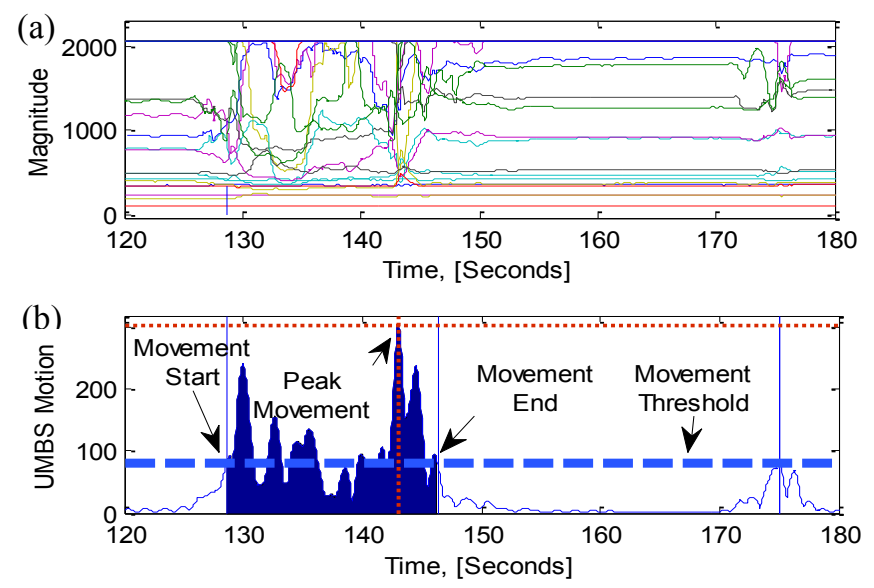

Figure 4. (a) Typical UMBS data during a movement. (b) UMBS motion metric derived from active UMBS data; movement start and end times are shown (a refractory period is employed).

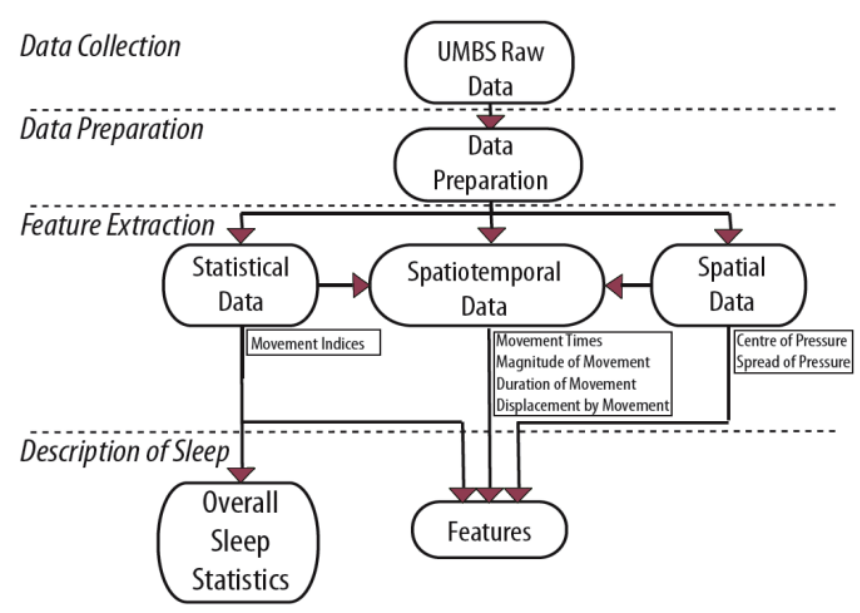

Figure 5. UMBS feature extraction flow chart 
Table I. Derived Nocturnal Movement Data

\begin{tabular}{cccc} 
& & Sub 1 & Sub 2 \\
\hline \hline \multirow{4}{*}{ Statistical } & Restlessness (\%) & & \\
& Small & 10.8 & 9.16 \\
& Medium & 2.35 & 4.05 \\
& Large & 4.32 & 4.26 \\
\hline \multirow{4}{*}{ Spatial } & ML Change (\%) & & \\
& $<0.1$ Tactels & 99.62 & 99.71 \\
& $0.5>$ Tactels $\geq 0.1$ & 0.358 & 0.27 \\
& $1>$ Tactels $\geq 0.5$ & 0.014 & 0.007 \\
& $\geq 1$ Tactels & 0.001 & 0.002 \\
\hline \multirow{4}{*}{ Spatiotemporal } & Duration & & \\
& $($ no. movements) & & \\
& $<1$ Sec & 15 & 8 \\
& $5>\operatorname{Sec} \geq 1$ & 40 & 31 \\
& $10>\operatorname{Sec} \geq 5$ & 18 & 12 \\
& $\geq 10$ Sec & 25 & 16 \\
\hline \hline
\end{tabular}

\section{DISCUSSION AND CONCLUSIONS}

This paper describes a non-contact method of sleep monitoring suitable for real-world and clinical settings. It derives movement features from a participant's time-in-bed. It extracts general statistics relating to bed-restlessness over the whole night and also quantifies the in-bed-motion throughout the night. Methods of extracting spatial variations in posture and a spatiotemporal quantification for each movement are also discussed. For example, this system has the ability to discriminate between prolonged low intensity movements and sudden high intensity movements.

Previous research has reported high agreement rates in the temporal discrimination of movement/non-movement monitoring using the UMBS compared to wrist actigraphy [12]. In addition to this, the calculation of quantitative statistics of nocturnal restlessness from the UMBS deployed in a real-world setting has also been previously reported upon [10]. This paper expands on this by taking advantage of the UMBS and analysing the movements spatially throughout the night. Collectively, the statistical, spatial and spatiotemporal descriptions of the nocturnal movements provide a more tangible description of in-bed-movement compared to wrist actigraphy, air-cells and RF motion sensors $[9,13,14]$.

A larger and more varied cohort will present a more coherent description of the variation of in-bed-movement amongst healthy adults, older adults and relatively healthy adults. This validation is currently underway. Future research will investigate the inferring of sleep quality, the detection of sleep apnoeas through the examination of the small body movements and the estimation of sleep and wakefulness augmenting the approach currently used in wrist actigraphy.

\section{ACKNOWLEDGMENT}

The authors wish to thank the clinic staff at Peamount Hospital for their help with data collection. They would also like to thank Adrian Burns, Julie Behan, Barry Greene, Michael McGrath and Terrance O'Shea of the Digital Health Group, Intel Ireland, for their advice and support.

\section{REFERENCES}

[1] F.P. Cappuccio, D. Cooper, L. D Elia, P. Strazzullo, and M.A. Miller, "Sleep duration predicts cardiovascular outcomes: a systematic review and meta-analysis of prospective studies.," European heart journal, Feb. 2011.

[2] K. Manabe, T. Matsui, M. Yamaya, T. Sato-Nakagawa, N. Okamura, H. Arai, and H. Sasaki, "Sleep patterns and mortality among elderly patients in a geriatric hospital.," Gerontology, vol. 46, pp. 318-22.

[3] L.E. Miles and W.C. Dement, "Sleep and Aging," Sleep, vol. 3, 1980, pp. 119-220.

[4] S. Ancoli-Israel, "Sleep problems in older adults: putting myths to bed," Geriatrics, vol. 52, Jan. 1997, pp. 20-30.

[5] 2003 National Sleep Disorders Research Plan, 2003.

[6] R.J. Rantz, M., Skubic, M., Koopman, L.J. Phillips, R.D. Guevara, and S.J. Miller, with G.L., "Evolution of an Early Warning System to Monitor Frail Elders in Independent Living," Journal of Healthcare Engineering, vol. 2, 2011, pp. 259-286.

[7] A. Rechtschaffen and A. Kales, "A manual of standardized terminology, techniques and scoring system for sleep stages of human subjects.," NIH Publication No. 204, US Government Printing Office, Washington, DC.,, 1968.

[8] H. Danker-Hopfe, P. Anderer, J. Zeitlhofer, M. Boeck, H. Dorn, G. Gruber, E. Heller, E. Loretz, D. Moser, S. Parapatics, B. Saletu, A. Schmidt, and G. Dorffner, "Interrater reliability for sleep scoring according to the Rechtschaffen \& Kales and the new AASM standard.," Journal of Sleep Research, vol. 18, 2009, p. 74.

[9] D.J. Mullaney, D.F. Kripke, and S. Messin, "Wristactigraphic estimation of sleep time," Sleep, vol. 3, 1980, pp. 83-92.

[10] L. Walsh, S. McLoone, J. Behan, and T. Dishongh, "The deployment of a non-intrusive alternative to sleep/wake wrist actigraphy in a home-based study of the elderly," Proc. 30th Annual International Conference of the IEEE Engineering in Medicine and Biology Society EMBS 2008, 2008, pp. 1687-1690.

[11] A. Camurri, "Recognizing emotion from dance movement: comparison of spectator recognition and automated techniques," International Journal of HumanComputer Studies, vol. 59, Jul. 2003, pp. 213-225.

[12] J. Behan, D. Prendergast, L. Walsh, and B. Quigley, "Social Rhythms and Nocturnal Routines in Community Dwelling Older Adults," ICOST, 2008, pp. 73-80.

[13] D.C. Mack, J.T. Patrie, P.M. Suratt, R.A. Felder, and M.A. Alwan, "Development and preliminary validation of heart rate and breathing rate detection using a passive, ballistocardiography-based sleep monitoring system.," IEEE Trans Inf Technol Biomed, vol. 13, Jan. 2009, pp. 111-120.

[14] P. de Chazal, E. O Hare, N. Fox, and C. Heneghan, "Assessment of sleep/wake patterns using a non-contact biomotion sensor," Engineering in Medicine and Biology Society, 2008. EMBS 2008. 30th Annual International Conference of the IEEE, 2008, pp. 514-517. 